\title{
Gerenciamento de Processos com a utilização de Workflow: automação dos processos de uma Instituição Comunitária De Ensino Superior
}

\section{Process Management with the use of Workflow: automation of the processes of a Community Institution of Higher Education}

\author{
Márcia Cristina Domingues Leite ${ }^{1}$ \\ Paula Rodrigues Scholant ${ }^{2}$ \\ Fábio Josende Paz ${ }^{3}$
}

\begin{abstract}
Resumo: A busca constante pela melhoria e inovação de seus processos administrativos faz com que cada vez mais Instituições de Ensino Superior incorporem metodologias e tecnologias que permitam o alcance dessas melhorias. Metodologias de gestão de processos em consonância com adventos como o da tecnologia permitem implementar mudanças significativas na rotina empresarial e garantindo eficiência no alcance dos objetivos. Sendo assim, o estudo teve como objetivo principal implantar um Workflow em uma Instituição Comunitária de Ensino Superior (ICES) na cidade de Bagé/RS e medir sua eficácia. Através de uma pesquisa descritiva e exploratória, foi realizado um estudo de caso na Universidade, onde foi implementado o Sistema de Protocolo Online no intuito de automatizar os processos administrativos. Alguns resultados já foram obtidos, como por exemplo, 40 serviços ativos podendo ser solicitados via sistema, em 30 dias mais de 200 protocolos abertos tramitando nos mais diversos setores. Nesta primeira etapa do estudo já foi possível identificar possíveis gargalos no fluxo de processos, métricas de tempo de espera de um protocolo por setor, entre outros. Acredita-se que, através do uso da ferramenta será possível propor melhorias, bem como um aprofundamento da técnica disponibilizando mais serviços através do sistema, automatizando tantos processos quanto for possível.
\end{abstract}

Palavras-chave: Automação; Gestão de Processos; Protocolos On-line

Abstract: The constant search for improvement and innovation of its administrative processes makes more and more Higher Education Institutions incorporate methodologies and technologies that allow the achievement of these improvements. Process management methodologies in line with technological advances allow us to implement significant changes in the business routine and guarantee efficiency in achieving the objectives. Thus, the main objective of the study was to implement a Workflow in a Community Institution of Higher Education (ICES) in the city of Bagé / RS and measure its effectiveness. Through a descriptive and exploratory research, a case study was carried out at the University, where the Online Protocol System was implemented in order to automate administrative processes. Some results have already been obtained, such as 40 active services that can be requested via the system, in

1Bacharel em Sistemas de Informação, Pós-graduanda em Informática na Educação - URCAMP, Campus Bagé - Av. Tupy Silveira, 2099 - Bagé (RS) - Brasil

\{marcialeite@urcamp.edu.br\}

2Bacharel em Administração de empresas URCAMP, Campus Bagé - Av. Tupy Silveira, 2099 - Bagé (RS) Brasil

\{paulascholant@urcamp.edu.br\}

3Mestre em Sistemas e Processos Industriais URCAMP, Campus Bagé - Av. Tupy Silveira, 2099 - Bagé (RS) - Brasil

\{fabiopaz@urcamp.edu.br\}

Rev. CCEI - URCAMP, V.23, n38, 2018 ( Submetido 10/09/2018; Aceito 04/10/2018)

https://doi.org/10.30945/ccei-v23i38.2663 
30 days more than 200 open protocols being processed in the most diverse sectors. In this first stage of the study it was already possible to identify possible bottlenecks in the process flow, metrics of waiting time of one protocol per sector, among others. It is believed that through the use of the tool it will be possible to propose improvements as well as a deepening of the technique making more services available through the system, automating as many processes as possible.

Keywords: Automation; Processes management; Online Protocols

\section{INTRODUÇÃO}

Para que uma Instituição de Ensino Superior (IES) consiga desempenhar seu papel perante a comunidade em que está inserida, se faz fundamental a busca pela melhoria e inovação de seus processos administrativos (PEREIRA et al., 2017). Santos (2014) esclarece que um processo é um conjunto de atividades dinamicamente coordenadas para entregar valor ao cliente final.

Nesse contexto, a gestão de processos tem um papel fundamental nas organizações e principalmente na sua cultura, pois ela determina a melhor forma com que as tarefas serão executadas tanto que já é vista como imprescindível na busca do alcance dos objetivos (VIEIRA, 2015). No âmbito das IES, Dias e Nunes (2017) destacam a necessidade iminente destas no que diz respeito à modernização de seus processos, sistematizando suas informações e políticas e garantindo uma maior eficiência no alcance de seus objetivos.

Para tanto, se faz necessário o mapeamento e a avaliação dos processos existentes na busca por determinar as melhorias necessárias e cabíveis e os impactos provenientes destas. Uma das áreas capazes de propor melhorias que irão atender à essas demandas é a Tecnologia da Informação (TI), pois ela viabiliza automações totais ou parciais dentro do fluxo de processos, capazes de facilitar as atividades de modo a oferecer vantagens tanto do ponto de vista humano (familiaridade, satisfação e aumento da produtividade) como administrativo (eficiência e eficácia). Aalst e Hee (2004) definem esta automatização de processos como Workflow net ao passo que, de acordo com os autores, através do Gerenciamento de Processos de Negócio, do inglês Business Process Management (BPM) aliado à práticas como Workflow net tem sido possível verificar um aumento considerável da produtividade nas organizações (AALST, 2010).

Os Sistemas de Informação (SI) adotados pelas organizações vão de encontro a esta demanda que permite uma correta modelagem do fluxo das atividades, aumentando a 
produtividade e reduzindo custos de forma significativa (NOCE, 2017).

A partir de tais premissas o estudo teve como objetivo implantar um Workflow em uma Instituição Comunitária de Ensino Superior (ICES) na cidade de Bagé/RS e medir sua eficácia. Tal ação visou a automação dos processos administrativos da referida instituição na busca por qualificar o fluxo da informação, dinamizando a execução das tarefas para melhor atender o consumidor final.

O estudo foi realizado pelo setor de Assessoria de Desenvolvimento Organizacional (ADO) da referida instituição e para o alcance do objetivo foi necessário inicialmente priorizar os processos mais utilizados pela instituição através da utilização da Matriz GUT, que de acordo com Bastos (2014) serve justamente orientar decisões mais complexas definindo prioridades de forma racional. Na sequência realizou-se o mapeamento dos processos priorizados juntamente com todos os setores envolvidos. Essa sequência de etapas pode ser melhor visualizada no trabalho publicado no Simpósio Brasileiro de Sistemas de Informação (SCHOLANT e PAZ , 2018).

Decorrida esta etapa, foi feita a configuração do processo no Workflow e do Redmine - este primeiro se trata de um software capaz de organizar os processos de trabalho, permitindo consulta a outras bases de dados institucionais e que alinha tais informações a indicadores de gestão. A aplicação permite que os processos antes feitos de forma física sejam abertos, tratados e encerrados digitalmente.

Resultados parciais já foram obtidos, com alguns serviços já sendo executados através da ferramenta envolvendo alguns setores da ICES, bem como o apontamento de possíveis gargalos de processos. Nas seções posteriores foram transcritos respectivamente o referencial teórico que embasou a ideia central do trabalho, trazendo a contribuição de outros autores que corroboram com os conceitos necessários para a realização deste. Na seção metodológica serão descritos todos os passos utilizados para realização do estudo e na sequência os primeiros resultados obtidos com a utilização da ferramenta. Finalmente, na seção de considerações finais foi possível traçar algumas considerações importantes este estudo e para estudos futuros.

\section{REFERENCIAL TEÓRICO}

Devido às transformações aceleradas e a acirrada competitividade no mundo corporativo, a fim de superar os inúmeros desafios de um cenário em mutação, a compreensão 
e a prática da inovação são imprescindíveis, uma vez que é por meio de atitudes inovadoras que é possível ampliar, reestruturar e aperfeiçoar as ações nos mais variados tipos de organizações (POSSOLLI, 2012).

Segundo De Sordi (2014), mudança é alterar um ambiente, estrutura, tecnologia ou pessoas de uma organização. Quando se diz respeito às inovações organizacionais é importante ressaltar que possuem caráter estritamente administrativo, envolvendo a gestão de pessoas e a gestão de estratégias para que os resultados almejados sejam alcançados com êxito.

As Instituições de Ensino Superior (IES) por serem organizações complexas que envolvem processos administrativos e acadêmicos apresentam dificuldades em atender todas as necessidades e expectativas de seus clientes, que desejam qualidade tanto em atendimento e quanto em ensino. Portanto, buscar novas formas eficientes e eficazes que provoquem mudanças aumentando a sua capacidade competitiva é de extrema importância (SALGADO, 2013).

Dentro da gestão estratégica encontra-se a gestão de processos que visa a empresa como um sistema aberto, onde os agentes interagem entre si e com o meio, em uma relação de interdependência. Conhecida como Business Process Modeling (BPM), a gestão de processos de negócio possui abordagem disciplinada capaz de identificar, desenhar, executar, medir, coordenar e controlar os processos de negócio, na busca de resultados que estejam alinhados com os objetivos organizacionais (CBOK 2013). Palavras que definem a abordagem por processos são: integração, cooperação e transparência. As organizações que utilizam desta gestão estabelecem como objetivo o foco no cliente final, ou seja, buscam constantemente a otimização de processos para satisfação dos clientes internos e externos (LAGE JÚNIOR,2016).

Através da metodologia de gestão de processos é possível que as inovações tecnológicas sejam aplicadas as organizações através do Workflow, que segundo Silva (2017), é a interação entre os processos através de sistemas específicos para automatização das atividades realizadas no fluxos mapeados, no qual todas as informações são passadas de um participante para outro através de ações alinhadas às regras do sistema já pré definidas, podendo este processo estar sendo automatizado no todo ou em parte, existindo atividades e documentos que ainda sejam tratados manualmente.

Rev. CCEI - URCAMP, V.23, n38, 2018 ( Submetido 10/09/2018; Aceito 04/10/2018) 
Ainda tratando dos inúmeros processos realizados pelas ICES, não seria possível analisá-los todos simultaneamente, contudo, existem ferramentas utilizadas para a priorização de processos sendo uma delas a Matriz GUT, que segundo Marshall Junior (2010), implica na representação dos problemas através da quantificação buscando estabelecer prioridades a serem abordadas sendo analisados pelos aspectos de gravidade, urgência e tendência onde os que obtiverem maior pontuação são definidos como prioritários.

\subsection{TRABALHOS CORRELATOS}

Diversos tipos de abordagem são propostos no que concerne a utilização de sistemas workflow. O estudo de Usirono (2003) tratava da verificação do impacto desta utilização nas organizações tendo como foco a utilização de um estudo de casos múltiplos. Costa (2009) formulou uma metodologia de modelagem de processos no sentido de apoiar a automação dos mesmos através do workflow. Ao final, o trabalho foi validado em uma empresa prestadora de serviços no ramo de energia nuclear com vistas à utilização do workflow.

Lousã e Sarmento (2002) em seu estudo fizeram um aprofundamento dos conceitos de gestão de processos e seus diversos níveis de abordagem com ênfase nos sistemas workflow. Ao final buscaram os autores demonstraram de que forma a utilização desta metodologia apoiam a gestão do conhecimento e dos processos administrativo de uma instituição. O presente estudo demonstra na prática a utilização do sistema de workflow e os resultados obtidos apartir da utilização da metodologia.

\section{METODOLOGIA}

A presente seção permitiu descrever todas as etapas necessárias para realização do estudo desenvolvido. Primeiramente foi realizada uma pesquisa da bibliografia cujo o caráter foi descritivo e exploratório e se buscou através de outros autores o entendimento científico relevante ao trabalho. Esse tipo de pesquisa permite uma visão geral acerca dos fenômenos envolvidos, descrevendo suas características e estabelecendo relações entre as variáveis (GIL, 1999). Cabe destacar também que a pesquisa compreende um estudo de caso, pois se reporta a uma Instituição Comunitária de Ensino Superior, envolvendo diversos setores e colaboradores para a obtenção dos resultados (YIN, 2001).

O segundo passo envolveu a utilização da Matriz GUT, a priorização e mapeamento 
dos processos (SCHOLANT E PAZ, 2018). Na sequência, foi feita a configuração dos processos que foram transformados em serviços no sistema de workflow juntamente com o gerenciador de tarefas Redmine, este último um software livre que auxilia na gerência de projetos e oferece outras especificidades como configurações backend e do kanban, interface responsável pela visualização dos tickets de todos os processos em andamento nas mais diversas etapas. Estas configurações permitem que a equipe que administra o sistema acompanhe cada etapa do processo bem como identificação de gargalos, intervalo de tempo dos protocolos por setor através de Gráficos de Gantt, técnica responsável por permitir determinar a data de início e de término de cada um dos processos ( PRIKLADNICKI et al., 2007). Nesta etapa foram realizados todos os ajustes de configuração, como por exemplo papéis, tipos de tarefas, fluxo de trabalho e etc. Essa primeira etapa de configuração inicial que ocorre no Redmine foi demonstrada na Figura 1.

Figura 1: Tela de configuração do Redmine.

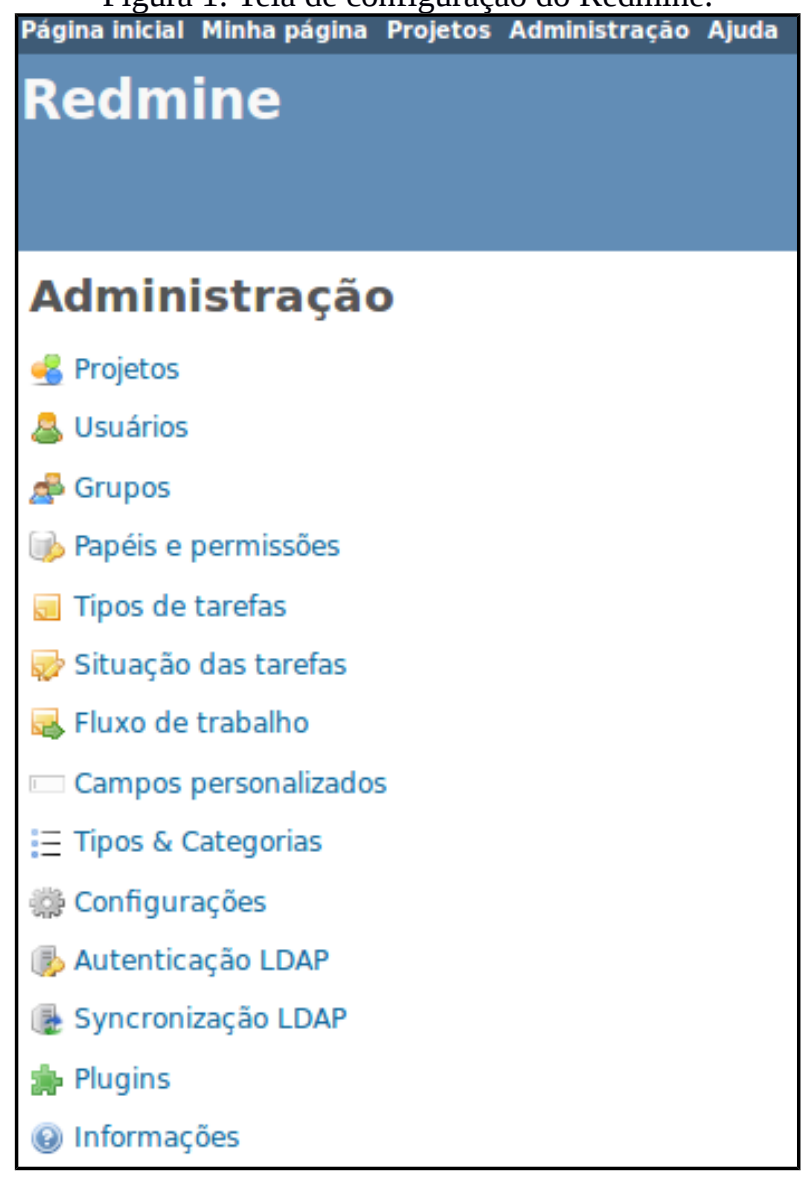

Fonte: Dados primários, 2018.

Na sequência foi feita a configuração na interface que foi disponibilizada aos 
colaboradores para que realizem a abertura, desenvolvimento e conclusão dos protocolos. Nessa etapa foram configurados serviços como os papéis que irão se envolver em cada um e as ações que fazem parte de cada processo. Também foram criadas as filas que serão disponibilizadas aos usuários de cada setor. Através destas filas cada colaborador saberá se existem protocolos abertos, pendentes e fechados, possibilitando um eficaz acompanhamento do fluxo. A Figura 2 demonstra a configuração de serviço, seguida da Figura 3 que demonstra todas essas configurações convertidas em uma interface gráfica que será utilizada pelos usuários do sistema.

Figura 2: Tela de configuração de Serviços no workflow

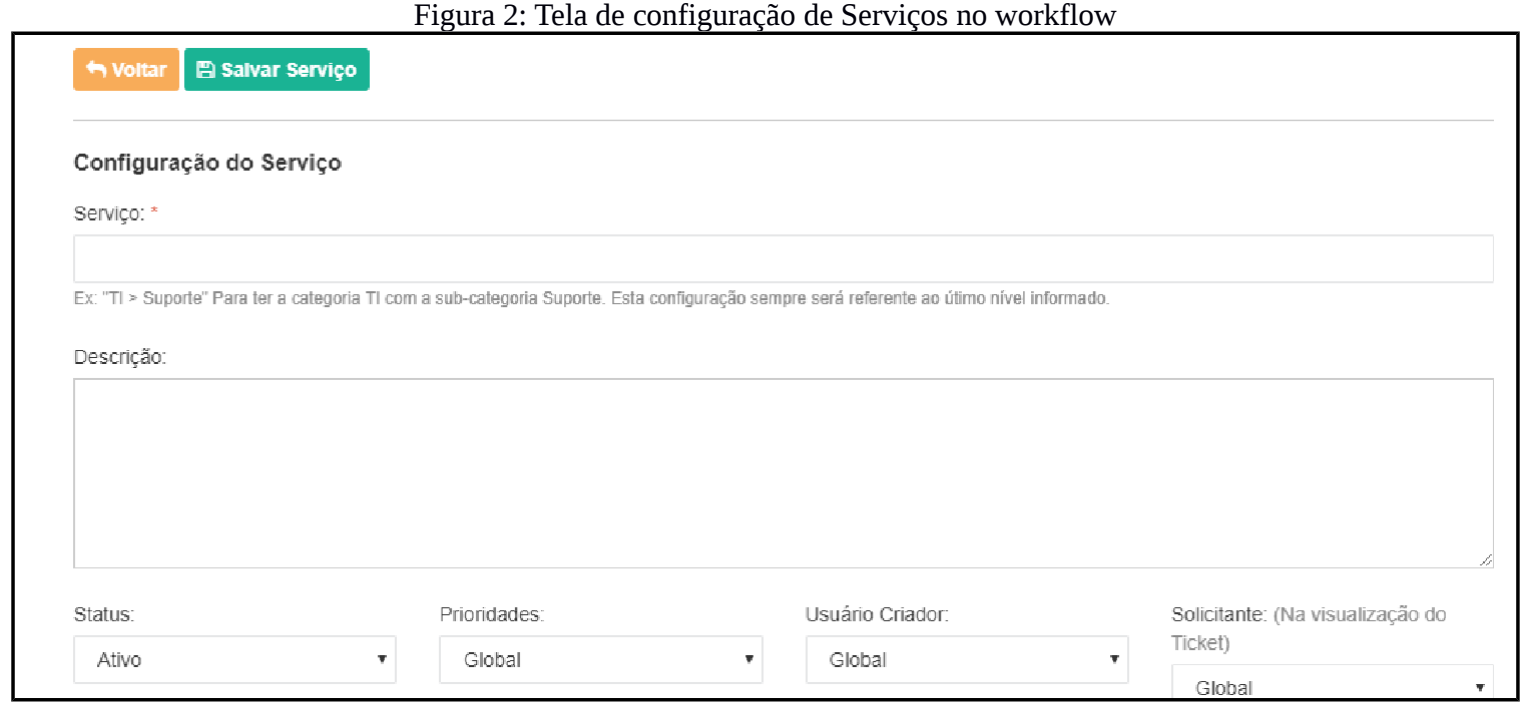

Fonte: Dados primários (2018).

Figura 3: Interface do usuário no workflow.

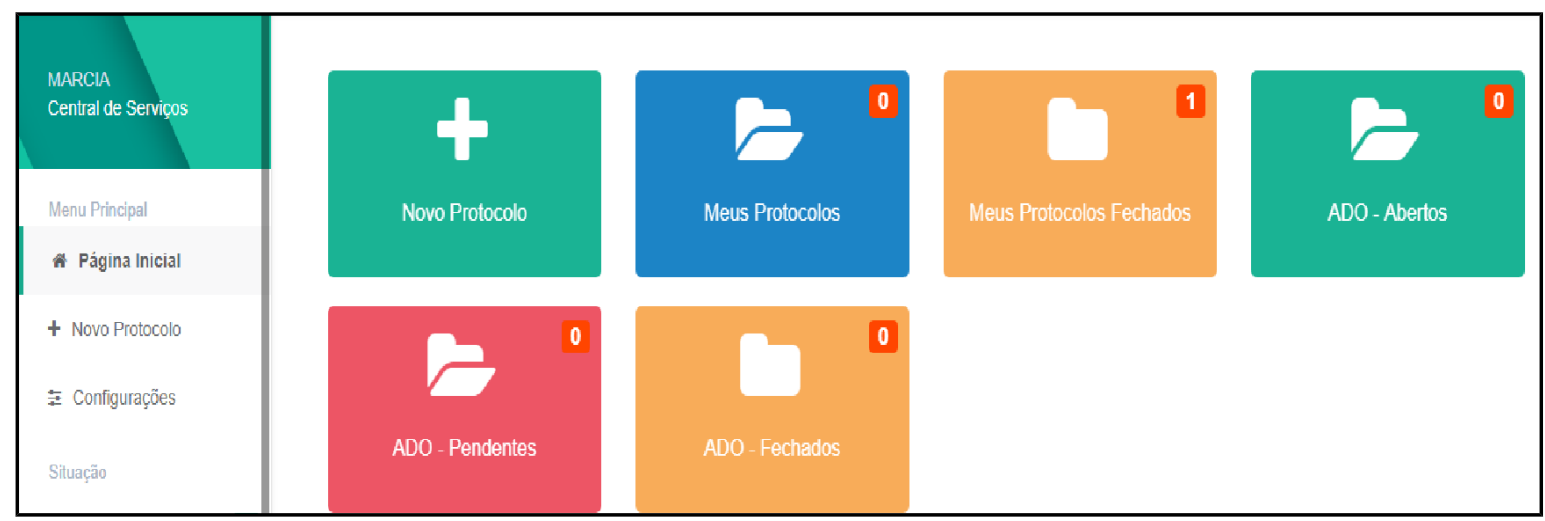

Fonte: Dados primários (2018)

Feito isso, o próximo passo foi socializar e treinar a equipe de colaboradores que farão uso do sistema. Foram disponibilizados manuais aos usuários, com o passo a passo de todas as possíveis ações a serem realizadas no sistema. O processo de sensibilização dos colaboradores 
também ocorre no sentido de demonstrar a importância da utilização do sistema, incorporação do acesso à rotina de trabalho e da não aceitação de processos físicos para os serviços já disponíveis via Protocolo Online.

\section{RESULTADOS}

O presente estudo visou implantar o sistema de workflow nos processos administrativos de uma ICES possibilitando transcorrer sobre alguns resultados obtidos. Atualmente 40 tipos de processos institucionais já podem ser solicitados através da aplicação e destes serviços ativos, 31 são abertos pela Central do Aluno, setor que recebe a maior parte dos alunos da Universidade. A Figura 5 demonstra parte dos protocolos ativos no sistema Redmine.

Figura 5: Serviços ativos Sistema Redmine

\begin{tabular}{|c|c|c|c|}
\hline & Abertas & Fechadas & Total \\
\hline Devolução de numerários & 1 & 9 & 10 \\
\hline Demanda Reitoria & 10 & 41 & 51 \\
\hline Quebra de protocolo & 0 & 0 & 0 \\
\hline Solicitação Compras & 0 & 0 & 0 \\
\hline $\begin{array}{l}\text { Auxílio } \\
\text { Mestrado/Doutorado }\end{array}$ & 0 & 1 & 1 \\
\hline $2^{\circ}$ via Diploma & 0 & 7 & 7 \\
\hline Bolsa funcionario/dep FP & 0 & 0 & 0 \\
\hline Requerimento de vaga & 0 & 0 & 0 \\
\hline Requisição de compras & 0 & 0 & 0 \\
\hline Demanda FAT & 0 & 0 & 0 \\
\hline Demanda PROCJUR & 2 & 0 & 2 \\
\hline Diária Reitoria & 0 & 10 & 10 \\
\hline $\begin{array}{l}\text { Reserva de veículo } \\
\text { Reitoria }\end{array}$ & 0 & 0 & 0 \\
\hline Hospedagem Reitoria & 0 & 2 & 2 \\
\hline $\begin{array}{l}\text { Devolução de Numerário } \\
\text { FIES }\end{array}$ & 13 & 1 & 14 \\
\hline Combustível Reitoria & 0 & 1 & 1 \\
\hline Declaração Sub judice & 0 & 0 & 0 \\
\hline Atestado de Concluinte & 0 & 3 & 3 \\
\hline $\begin{array}{l}\text { Atestado Provável } \\
\text { Concluinte }\end{array}$ & 0 & 5 & 5 \\
\hline Atestado de Vínculo & 0 & 2 & 2 \\
\hline Trancamento de Matrícula & 9 & 6 & 15 \\
\hline Solicitação de diária & 6 & 22 & 28 \\
\hline Ajuste de matrícula & 34 & 130 & 164 \\
\hline Parcelamento de dívida & 0 & 1 & 1 \\
\hline Curso de Nivelamento & 0 & 4 & 4 \\
\hline Ajuda de Custo & 0 & 2 & 2 \\
\hline Solicitação Financeira & 2 & 0 & 2 \\
\hline Solicitação Acadêmica & 0 & 1 & 1 \\
\hline
\end{tabular}

Fonte: Dados primários (2018)

Alguns aspectos importantes a respeito da abertura de protocolos através do sistema 
que podem ser destacados é o fato de que o requisitante ao realizar a abertura do chamado recebe um e-mail informando todas as atualizações realizadas no sistema pelos setores. Essa informação permite que o solicitante de algum serviço esteja constantemente sendo informado sobre o diligenciamento de sua demanda. Outro detalhe importante é que todos os setores envolvidos em determinado serviço conseguem visualizar em suas filas os processos existentes. Isso garante uma eficiente comunicação entre os setores, otimizando o fluxo das tarefas.

Do ponto de vista gerencial, os primeiros resultados já podem ser analisados como segue:

- Obtenção de indicadores de produtividade;

- Identificação de gargalos nos processos: nesse tópico podemos evidenciar o serviço de Ajuste de Matrícula que já apresenta um gargalo em seu fluxo, localizado no setor que cuida dos alunos beneficiados pelo FIES. Esse resultado é obtido através do Kanban disponibilizado pela ferramenta e que demonstra na tela do monitor o fluxo dos processo em tempo real. Na Figura 6 é possível verificar protocolos em vermelho que foram encaminhados ao setor FIES. A cor vermelha é indicativa de que o tempo configurado no SLA (ver explicação no tópico 3 desta seção) foi excedido.

Figura 6: Kanban de acompanhamento de protocolos.

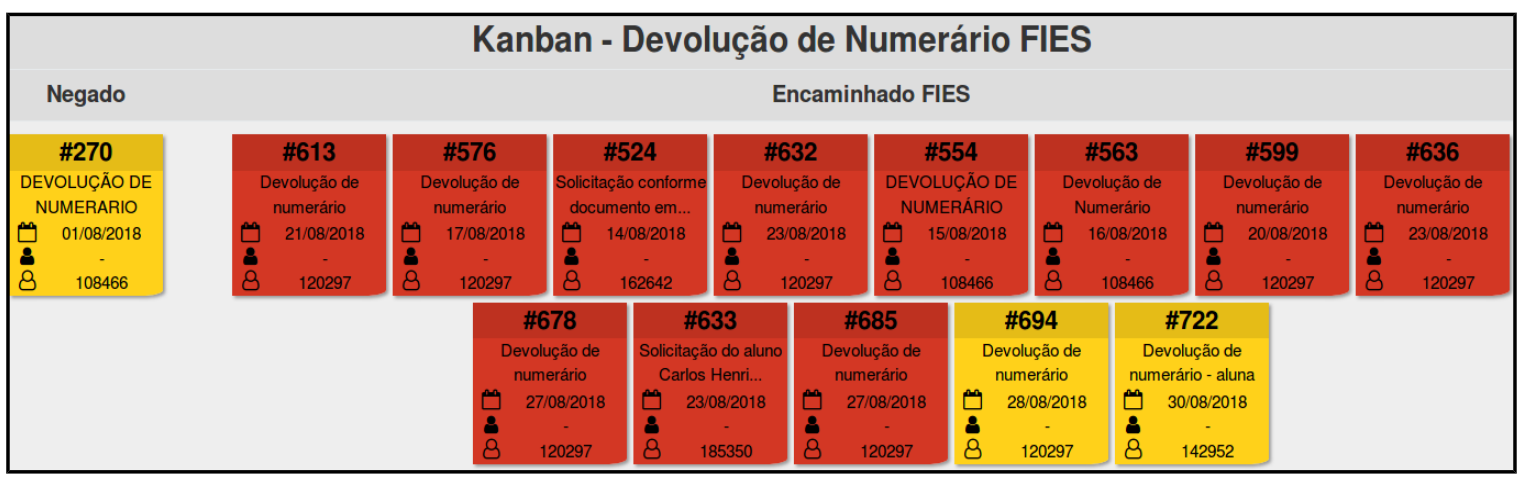

Fonte: Dados primários (2018).

Ao verificar o gargalo, a gestão foi notificada e solicitou um aprimoramento do processo e também um treinamento mais efetivo aos integrantes do setor FIES.

Média do tempo de finalização dos processos: com o uso da ferramenta é possível quantificar o tempo em que cada protocolo aberto esteve em determinado 
setor. Utilizou-se como exemplo o serviço de “Devolução de numerários” onde foram analisados todos os protocolos abertos e verificados o número de horas em que cada um esteve em um determinado setor. Posteriormente foi feita a média do somatório pelo número de setores em que este passou. O resultado foi convertido em dias, onde foi obtida a média de 2,79 que revela que cada protocolo leva em média 03 dias para ser concluído, o que mostra que o $\mathrm{SLA}^{4}$ de 02 dias por etapa do processo, configurados no sistema difere do tempo que os setores levam para resolver suas demandas. Cabe destacar que antes da implementação do workflow a média de conclusão de cada processo, do primeiro setor até a sua conclusão era de 30 dias. A Tabela 1 demonstra de forma detalhada como foram tabulados os dados descritos.

Tabela 1: Tabulação e análise do tempo do serviço por setor.

\begin{tabular}{|c|c|c|c|c|c|c|c|}
\hline \multirow{2}{*}{$\begin{array}{c}\text { ID } \\
\text { PROTOCOLO }\end{array}$} & PROAD & PROAC & PROIPPEX & TESOURARIA & SOMA & MÉDIA & MÉDIA EM DIAS \\
\cline { 2 - 8 } & PEMPO POR SETOR EM HORAS & & \\
\hline 241 & 140 & 0 & 0 & 0 & 140 & 70 & 2,9 \\
\hline 170 & 46 & & & 98 & 144 & 77 & 3,3 \\
\hline 540 & 23,5 & & & 138 & 161,5 & 80,75 & 3,3 \\
\hline 517 & 1 & & & & 1 & 1 & 0,04 \\
\hline 407 & 95 & & & 24 & 119 & 59,5 & 2,5 \\
\hline 354 & 40,5 & & 110 & 17 & 167,5 & 55,83 & 2,33 \\
\hline 332 & 57 & & & 0,2 & 57,2 & 28,6 & 1,2 \\
\hline 314 & 208 & & & 96 & 304 & 152 & 6,3 \\
\hline 305 & 3 & & & 141 & 144 & 77 & 3,3 \\
\hline
\end{tabular}

Fonte: Dados primários (2018)

O processo de Devolução de numerário é aberto pelo aluno, professor ou funcionário na Central do Aluno e encaminhado para a PROAD (Pró-Reitoria Administrativa). Esta por sua vez, ao fazer a verificação financeira da solicitação, tem como opções solicitar informações na PROAC ${ }^{5}$ ou na PROIPPEX ${ }^{6}$. Após receber tais informações, a PROAD Nega ou Encaminha para Tesouraria para que o pagamento seja efetuado. Ao receber o protocolo na Tesouraria, os atendentes realizam a quitação e concluem o protocolo. Este fluxo pode ser 4 SLA (do inglês Service Level Agreement) é um acordo de nível de serviço, em outras palavras serve, dentre outros, para monitorar o tempo em que cada protocolo permaneceu em cada setor. O SLA é um indicador previamente configurado com o tempo estimado e, no caso deste tempo ser excedido, ele faz com que o ticket do Kanban fique vermelho, sugerindo protocolos pendentes a mais tempo do que se espera (SLEDGIANOWSKI;

LUFTMAN, 2005)

5 Pró-Reitoria Acadêmica.

6 Pró-Reitoria de Inovação, Pesquisa e Extensão. 
melhor visualizado na Figura 7.

Figura 7: Diagrama do processo de Devolução de Numerário.

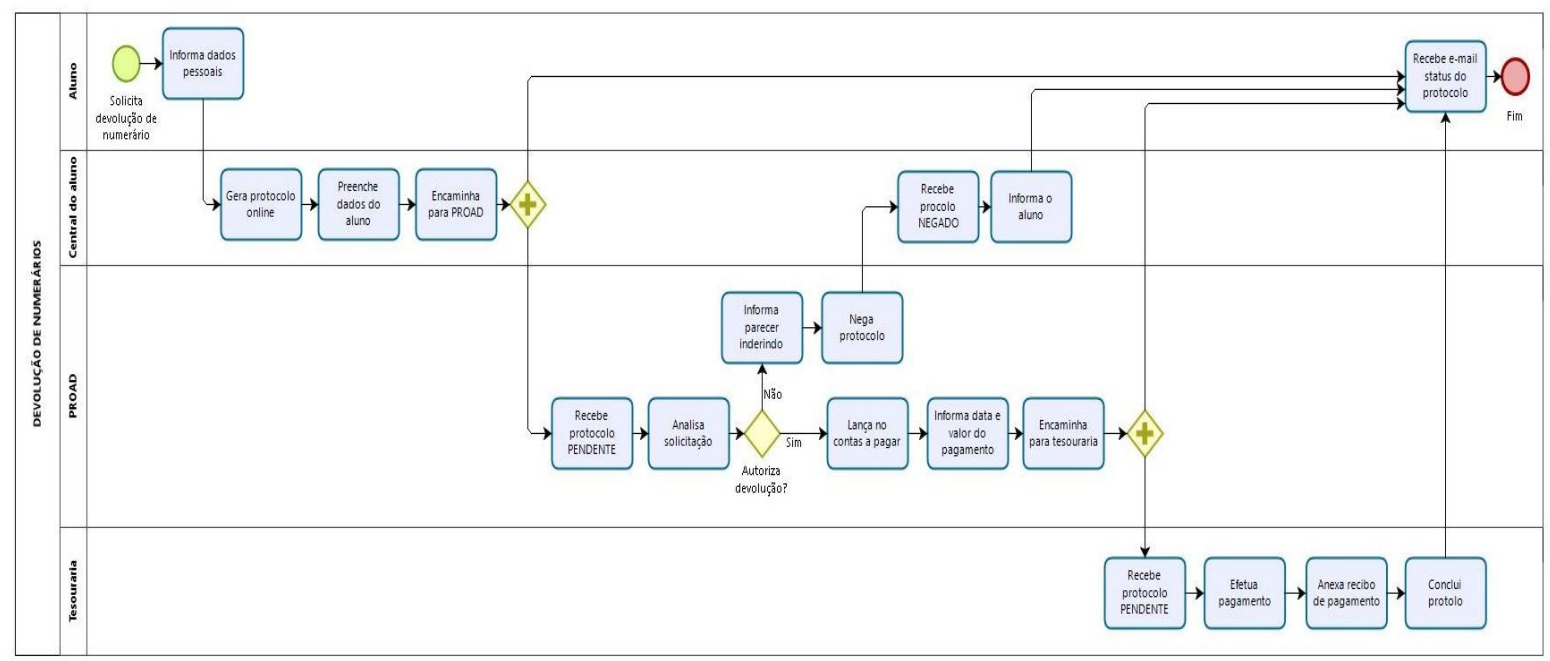

Fonte: Bizzagi Modeler (2018)

O fato da implantação ainda estar em seu estágio inicial limita o trabalho no sentido de que ainda não foi possível realizar a avaliação da usabilidade com os usuários do sistema bem como os impactos organizacionais dessa implementação. Entretanto, já foi possível a obtenção de algumas métricas que auxiliam na tomada de decisões no sentido de otimizar o uso do sistema workflow. Destacaram-se nessa quantificação, erros cometidos pelos usuários do sistema onde foram identificados problemas com documentação faltante e troca de documentação. Acredita-se que tais erros operacionais se deviam pela falta de conhecimento do processo por parte de alguns colaboradores. Por esse motivo foi feita uma nova etapa de treinamentos com foco nos colaboradores que tiveram maior número de erros no uso do sistema.

No que se refere à resultados financeiros, podemos tomar como exemplo o protocolo de Solicitação de Diárias que permite mensurar o valor gasto de diárias para eventos, treinamentos, visitas técnicas e etc. Quanto à melhoria no fluxo das informações é possível tomar como exemplo a solicitação de uma demanda para um campus de outra cidade. Este trâmite era feito pelo uso de malotes semanais que eram analisados e só então resolvidos; com o uso do sistema de workflow, essa logística é feita de forma mais rápida o que permite deduzir um aumento no índice de satisfação do aluno ao receber respostas de seus protocolos 
mais rapidamente.

Estudos futuros prospectam a avaliação da usabilidade do sistema e a continuidade em questões como treinamento de equipe como forma de facilitar a utilização da ferramenta. Também projeta-se a implementação do sistema para os demais Câmpus da instituição.

\section{CONSIDERAÇÕES FINAIS}

O trabalho ora apresentado se mostrou importante no que diz respeito à utilização de sistemas de Workflow como ferramenta para auxiliar na gestão de processos institucionais.

A aplicabilidade da Matriz GUT se mostrou capaz de facilitar a priorização de processos críticos e seu uso juntamente com o Gerenciamento de Processos através da metodologia BPM, resultou no correto mapeamento dos processos para utilização do sistema de Workflow ora apresentado. A utilização do software permitiu a obtenção dados gerenciais relevantes para a tomada de decisões.

Vários processos foram melhorados com a implantação do sistema de workflow, acredita-se que isso ocorra pelo fato da melhor organização e simplificação do fluxo de informações ocasionadas pela automatização dos mesmos. Acredita-se que a integração entre metodologias gerenciais e automações tecnológicas promovem mudanças significativas na rotina administrativa das instituições, o que permite sugerir para estudos o aprofundamento do uso de tais soluções, oportunizando importantes melhorias nos mais diversos segmentos da instituição. Os processos continuam sendo implantados e em breve a integração no portal do aluno o que permitirá que o aluno abra seu protocolo diretamente de seu celular, tablet ou computador. Para trabalhos futuros será avaliada a usabilidade do Workflow bem como a mensuração da satisfação do usuário, além da implantação de indicadores gerenciais. Embora a interface já apresente um design responsivo, planeja-se também a versão mobile da aplicação. Como limitações pode-se dizer que o pouco tempo de utilização do sistema não permitiu apresentar mais números de atendimentos e médias.

\section{AGRADECIMENTOS}

Este trabalho contou com financiamento da URCAMP através da Pró-Reitoria de Inovação, Pós-Graduação, Pesquisa e Extensão, por meio de bolsa de Iniciação Científica. 


\section{REFERÊNCIAS}

AALST, W.; HEE, K. Workflow management: Models, methods, and systems. The MIT Press Cambridge, Massachusetts. London, England, 2004.

AALST, W. M. P. van der. Modern business process automation. In: HOFSTEDE, A.

H. M. (Ed.), p. 3 a 19, 2010.

BASTOS, M. Ferramentas da Qualidade - Matriz Gut, 2014. Disponível em <https://goo.gl/sQM1dr >. Acesso em 22/08/2018.

COSTA, L. Formulação de uma metodologia de modelagem de processos de negócio para implementação de workflow. Dissertação de Mestrado em Engenharia da Computação Universidade Federal do Paraná Campus Ponta $\quad$ Grossa, 130p. $2009 . \quad$ Disponível em: < http://www.pg.utfpr.edu.br/dirppg/ppgep/dissertacoes/arquivos/112/Dissertacao.pdf>. Acesso em: 02/10/2018.

BPM CBOK. Guia para Gerenciamento de Processo de Negócio versão 3.0, 2013.

DE SORDI, J. O. Gestão por processos: uma abordagem da moderna administração. 4. Ed. São Paulo: Saraiva, 2014.

DIAS, M. A; NUNES, E. Mercado e educação: cenário atual do ensino superior brasileiro. REFAS - Revista Fatec Zona Sul. v.3, n.2, fevereiro 2017. Acesso em: 22/08/2018.

GIL, A. C. Métodos e técnicas de pesquisa social. 5. ed. São Paulo: Atlas, 1999.

LAGE JÚNIOR, M. Mapeamento de processos de Gestão empresarial. Curitiba, PR, 2016.

LOUSÃ, M. SARMENTO, A. Implementação de utilização de sistemas workflow como suporte à gestão do conhecimento: um estudo de caso. Atas da Conferência da Associação Portuguesa de Sistemas de Informação. ISSN 2183-489X. 2002. Disponível em: <http://capsi.apsi.pt/index.php/capsi/article/view/343/327>. Acesso em: $02 / 10 / 2018$.

SILVA, M. C. B. Metodologia para especificação de processos acadêmicos usando padrões de workflow e redes de Petri. 2017. 128 f. Dissertação, Mestrado em Modelagem Computacional de Conhecimento Universidade Federal de Alagoa/Ufal, 2017.

MARSHALL JUNIOR, I. Gestão da Qualidade. 10. Ed. - Rio de Janeiro: FGV,2010.

NOCE, F. S. Uma abordagem para a Modelagem, Analise e Simulacao de processos de negocios interorganizacionais baseada nas Workflow nets e na ferramenta CPN Tools. Disponível em: <https://goo.gl/HdjPbv >. Acesso em: 23/08/2018.

Rev. CCEI - URCAMP, V.23, n38, 2018 ( Submetido 10/09/2018; Aceito 04/10/2018) 
PEREIRA, F. L; JACOBSEN, A. L.; MARTINA, J. E.; LENGLER, F. R. A importância da inovação na gestão de processos administrativos da Universidade Pública, por meio da implementação da Tecnologia de Certificação Digital. Revista da UNIFEBE, ISSN 2177-742X, Brusque, v. 1, n. 21, mai/ago. 2017. Disponível em: $<$ http://brjd.com.br/index.php/BRJD/article/view/281>. Acesso em: 22/08/2018.

POSSOLI, G. E. Gestão da inovação e do conhecimento. Curitiba: InterSaberes, 2012.

PRIKLADNICKI, R.; ROSA, R.; KIELING, E. Ensino de Gerência de Projetos de Software com o Planager. XVIII Simpósio Brasileiro de Informática na Educação - SBIE - Mackenzie - 2007 Disponível em:

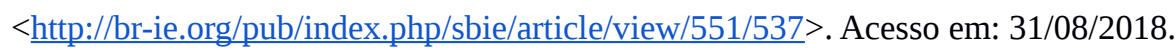

SALGADO, C. C. R. 2013. Contribuição à melhoria de processos organizacionais: Uma avaliação empírica sob a perspectiva de mapeamento de processos em uma Universidade Federal da Paraíba. Disponível em : $<$ https://doi.org/10.15628/holos.2013.1034>. Acesso em: 31/08/2018.

SCHOLANT, P.R., PAZ, F. J. Gestão de Processos e Tecnologia da Informação: Aplicação da Metodologia Business Process Management para Automatização de Processos . V Workshop de Iniciação Científica em Sistemas de Informação, Caxias do Sul, RS, 4 a 8 de Junho de 2018. Disoonível em:

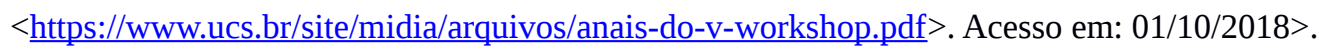

SLEDGIANOWSKI, D.; LUFTMAN, J. IT-business strategic alignment maturity: a case study. Journal of Cases on Information Technology, v. 7, n. 2, p. 102-120, 2005.

USIRONO, C. H. Tecnologia Workflow: $O$ impacto de sua utilização nos processos de negócio. Um estudo de casos múltiplos. Dissertação de mestrado em Administração. Universidade de São Paulo (USP), 2003, 178p. Disponível em: < $<$ ttp://www.teses.usp.br/teses/disponiveis/12/12139/tde-08122003-233842/en.php>. Acesso em: 02/10/2018.

VIEIRA, T. V. G. Mapeamento do processo de Alvarás de Construção, utilizando a metodologia de mapeamento de processos BPM. Tarso Vinicius Gianini Vieira, 2015.

YIN, R. K. Estudo de caso: planejamento e métodos. 2 ed. Porto Alegre: Bookman. 2001 